\title{
Shock transition to detonation in channels with obstacles
}

\author{
G.B. Goodwin, ${ }^{*}$ R.W. Houim, E.S. Oran
}

Department of Aerospace Engineering, University of Maryland, College Park, Maryland, 20742, USA

*Corresponding author: Gabriel Goodwin, ggoodwin@umd.edu

Colloquium: Detonations, Explosions and Supersonic Combustion

5730 words, Word Count Method 1

Main text: 2628 words

Nomenclature: 0 words

References: 472 words

Fig. 1: 124 words

Fig. 2: 457 words

Fig. 3: 641 words

Fig. 4: 152 words

Fig. 5: 220 words

Fig. 6: 126 words

Fig. 7: 132 words

Fig. 8: 161 words

Fig. 9: 184 words

Table 1: 410 words

Affirmation to pay color reproduction charges: We do not wish to pay color reproduction charges. Please use black and white in the print version and color in the online version. 


\begin{abstract}
Multidimensional numerical simulations of an unconfined, homogeneous, chemically reactive gas were used to study interactions leading to deflagration-to-detonation transition (DDT). The configuration studied was a long rectangular channel with regularly spaced obstacles containing a stoichiometric mixture of ethylene and oxygen, initially at atmospheric conditions and ignited in a corner with a small flame. The compressible reactive Navier-Stokes equations were solved by a high-order numerical algorithm on a locally adapting mesh. The initial laminar flame develops into a turbulent flame with the creation of shocks, shock-flame interactions, shock-boundary layer interactions, and a host of fluid and chemical-fluid instabilities. The final result may be eventual deflagration-to-detonation transition (DDT). Here two types of simulations are described, one with DDT occurring in a gradient of reactivity, which is common in the channels with higher obstacles, and another in which DDT arises from energy focusing as shocks converge. For the latter case, the rate of energy deposition necessary to initiate a detonation in the unburned gas is analyzed using a control volume analysis.
\end{abstract}

Keywords: DDT; Turbulent flame; Shock interactions; Direct initiation; Numerical simulations

\title{
1 Introduction
}

Deflagration-to-detonation transition (DDT) is a complex process involving turbulence, shock-flame interactions, shock collisions and reflections, and interactions of shocks with boundary layers. It is an important topic because DDT must be prevented in many cases $[1,2]$ and optimized for specific applications [3-5]. DDT may be initiated by a number of different mechanisms that have been seen in both experiments and computations [6-15]. Not all of these mechanisms, or the interplay among them, are fully understood.

Direct initiation of a detonation in a gas has been studied theoretically and experimentally [68]. Sufficiently large external energy addition at a timescale that is small relative to the acoustic timescale of the gas can result in a direct initiation of a detonation without first creating a deflagration [16]. Recently, the response of the gas to a localized, transient energy deposition has been studied by asymptotic analysis in an effort to quantify the gas response (deflagration or detonation) [17]. Initiation of a detonation through a gradient of chemical reactivity $[18,19]$ has been investigated extensively using numerical simulations, as summarized in [10]. DDT in channels with obstacles has been computed for a variety of fuel-oxidizer mixtures [11-13] and has been the subject of experimentation [20].

The effect of obstacles, often characterized by a blockage ratio $(b r$, or the obstacle height divided by the channel height), on flame acceleration and DDT has been investigated experimentally [14, 15] 
and numerically [21]. Gamezo et al. [21] noted the competing effects of high blockage ratios: larger obstacles promote non-uniform flow, which leads to fast flame acceleration and they also weaken shocks diffracting over large obstacles. These authors also observed that the location in the channel where DDT occurs does not vary significantly for $b r=0.31-0.56$ range, but it increases sharply outside of this interval for both higher and lower $b r$.

As a background to this paper, a series of simulations was performed with $b r=0.05-0.8$ in order to identify how the mechanism of DDT changes as blockage ratio decreases. The detailed results of these simulations, and some unusual trends noted, will be presented in a subsequent paper. In several of these simulations, however, shocks and fluid instabilities evolved that caused flame acceleration and transition to detonation due to energy focusing as opposed to the more predictable detonations that occur in Mach reflections against obstacles in systems with high br. Maeda et al. [22] saw similar phenomena in their experiments in channels with $b r=0.083$ and 0.25 . The purpose of this paper is to extract results for low $b r$ and compare the mechanism of DDT with those seen for the relatively high $b r=0.5$.

\section{Numerical and Physical Model}

The numerical model solves the full set of Navier-Stokes equations in unsteady, fully compressible, chemically reacting gas, as described in [10]. The reaction of a stoichiometric mixture of ethylene and oxygen, with specific ethylene-oxygen parameters courtesy of Alp Ozgen, is modeled using a simplified, calibrated chemical-diffusive model of the form,

$$
\frac{d Y}{d t} \equiv \dot{w}=-A \rho Y \exp \left(-E_{\mathrm{a}} / R T\right)
$$

where $\rho, T, Y$, and $\dot{w}$ are the mass density, temperature, mass fraction of reactant, and reaction rate, respectively. $A$ is the pre-exponential factor, and $E_{\mathrm{a}}$ is the activation energy. Thermal conductivity, $\kappa$, viscosity, $\nu$, and mass diffusivity, $D$, were modeled using values of $1.0 \times 10^{-5} \mathrm{~g} / \mathrm{s}-\mathrm{cm}-\mathrm{K}^{0.7}, 7.0 \times 10^{-6}$ $\mathrm{g} / \mathrm{s}-\mathrm{cm}-\mathrm{K}^{0.7}$, and $1.0 \times 10^{-5} \mathrm{~g} / \mathrm{s}-\mathrm{cm}-\mathrm{K}^{0.7}$, respectively. A Godunov algorithm, fifth-order accurate in space and third-order accurate in time [23], is used to solve the equations on a dynamically adapting grid [24]. The simulations described below used grids with minimum size $d x_{\min }=3.3 \mu \mathrm{m}$ and coarsest size ranging from $d x_{\max }=26.7$ to $53.3 \mu \mathrm{m}$. The largest grid had 60 cells across the channel height and the smallest had 960 cells. This choice of grids was tested and shown to resolve the flames, shocks, boundary layers, and other important flow and chemical structures.

The input parameters in Table 1 were chosen to reproduce the flame and detonation properties of the 
Table 1. Input model parameters and output combustion wave properties for stoichiometric ethylene and oxygen initially at $1 \mathrm{~atm}$ and $298 \mathrm{~K}$.

\begin{tabular}{lll}
\hline & \multicolumn{1}{c}{ Input } & \\
\hline$P_{\mathrm{o}}$ & $1 \mathrm{~atm}$ & Initial Pressure \\
$T_{\mathrm{o}}$ & $298 \mathrm{~K}$ & Initial Temperature \\
$\rho_{\mathrm{o}}$ & $1.27 \times 10^{-3} \mathrm{~g} / \mathrm{cm}^{3}$ & Initial Density \\
$\gamma$ & 1.2195 & Adiabatic Index \\
$M$ & $31 \mathrm{~g} / \mathrm{mol}$ & Molecular Weight \\
$A$ & $1.05 \times 10^{12} \mathrm{~cm}^{3} /(\mathrm{g} \mathrm{s})$ & Pre-Exponential Factor \\
$E_{\mathrm{a}}$ & $39.2 R T_{\mathrm{o}}$ & Activation Energy \\
$q$ & $59.7 R T_{\mathrm{o}} / \mathrm{M}$ & Chemical Energy Release \\
$\nu_{\mathrm{o}}$ & $7.0 \times 10^{-6} \mathrm{~g} / \mathrm{s}-\mathrm{cm}-\mathrm{K}^{0.7}$ & Viscosity \\
$\kappa_{\mathrm{o}}=D_{\mathrm{o}}$ & $1.0 \times 10^{-5} \mathrm{~g} / \mathrm{s}-\mathrm{cm}-\mathrm{K}^{0.7}$ & Transport Constants \\
\hline & & \\
\hline$S_{\mathrm{L}}$ & $\mathrm{Output}$ & \\
$T_{\mathrm{b}}$ & $413 \mathrm{~cm} / \mathrm{s}$ & Laminar Flame Speed \\
$\rho_{\mathrm{b}}$ & $11.7 T_{\mathrm{o}}$ & Post-Flame Temperature \\
$x_{\mathrm{l}}$ & $0.085 \rho_{\mathrm{o}}$ & Post-Flame Density \\
$D_{\mathrm{CJ}}$ & $8.88 \times 10^{-3} \mathrm{~cm}$ & Laminar Flame Thickness \\
$P_{\mathrm{ZND}}$ & $2.20 \times 10^{5} \mathrm{~cm} / \mathrm{s}$ & CJ Detonation Velocity \\
$P_{\mathrm{CJ}}$ & $54.5 P_{\mathrm{o}}$ & Post-Shock Pressure \\
$T_{\mathrm{ZND}}$ & $27.8 P_{\mathrm{o}}$ & Pressure at CJ Point \\
$T_{\mathrm{CJ}}$ & $6 T_{\mathrm{o}}$ & Post-Shock Temperature \\
$\rho_{\mathrm{ZND}}$ & $15.5 T_{\mathrm{o}}$ & Temperature at CJ Point \\
$\rho_{\mathrm{CJ}}$ & $9.8 \rho_{\mathrm{o}}$ & Post-Shock Density \\
$x_{\mathrm{d}}$ & $2 \rho_{\mathrm{o}}$ & Density at CJ Point \\
$\lambda$ & $3.2 \times 10^{-4} \mathrm{~cm}$ & Half-Reaction Thickness \\
\hline & $\sim 0.02 \mathrm{~cm}$ & Detonation Cell Size \\
\hline
\end{tabular}

ethylene-oxygen mixture initially at $298 \mathrm{~K}$ and $1 \mathrm{~atm}$. Using the input parameters in one-dimensional (1D) and two-dimensional (2D) solutions of the Navier-Stokes equations produces the output flame and detonation properties listed. To estimate the detonation cell height, $\lambda$, for this model, a 2D detonation was computed and an average was taken of cell sizes after an initially overdriven detonation had decayed to the Chapman-Jouguet velocity. The value $\lambda=0.2 \mathrm{~mm}$ agrees surprisingly well with reported experimental results, which range from $0.1 \mathrm{~mm}$ to $2 \mathrm{~mm}[25]$.

The geometrical setup is shown in Fig. 1. Obstacles are uniformly spaced along the wall. The obstacle width and height are constant through the channel. The $b r$ for the simulations discussed here are 0.5 and 0.1 . The channel is open at the right, with symmetry planes at the left and top boundaries. The bottom wall and all obstacle surfaces are no-slip adiabatic boundaries. To ignite the mixture, a circular region of hot, burned product at $3500 \mathrm{~K}$ and $1 \mathrm{~atm}$ is placed at the left wall. A channel height of $0.32 \mathrm{~cm}$ was chosen for several reasons. Experimental data for DDT in small channels with stoichiometric ethylene-oxygen, such as the work by Wu et al. [25], is available for comparison. The small scale detonation properties of this fuel-oxidizer mixture, such as small detonation cell sizes, fast reactions, and energy release, make ethylene-oxygen an ideal fuel-oxidizer mixture for studying DDT 
in small channels.

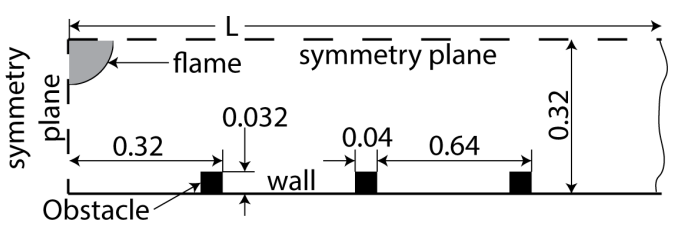

Fig. 1. Computational setup in units of $\mathrm{cm}$. Obstacles evenly spaced along channel length $L$. Radius of the initial burned region is $0.08 \mathrm{~cm}$. Blockage ratio is 0.1 in this case.

\section{Flame Acceleration and DDT}

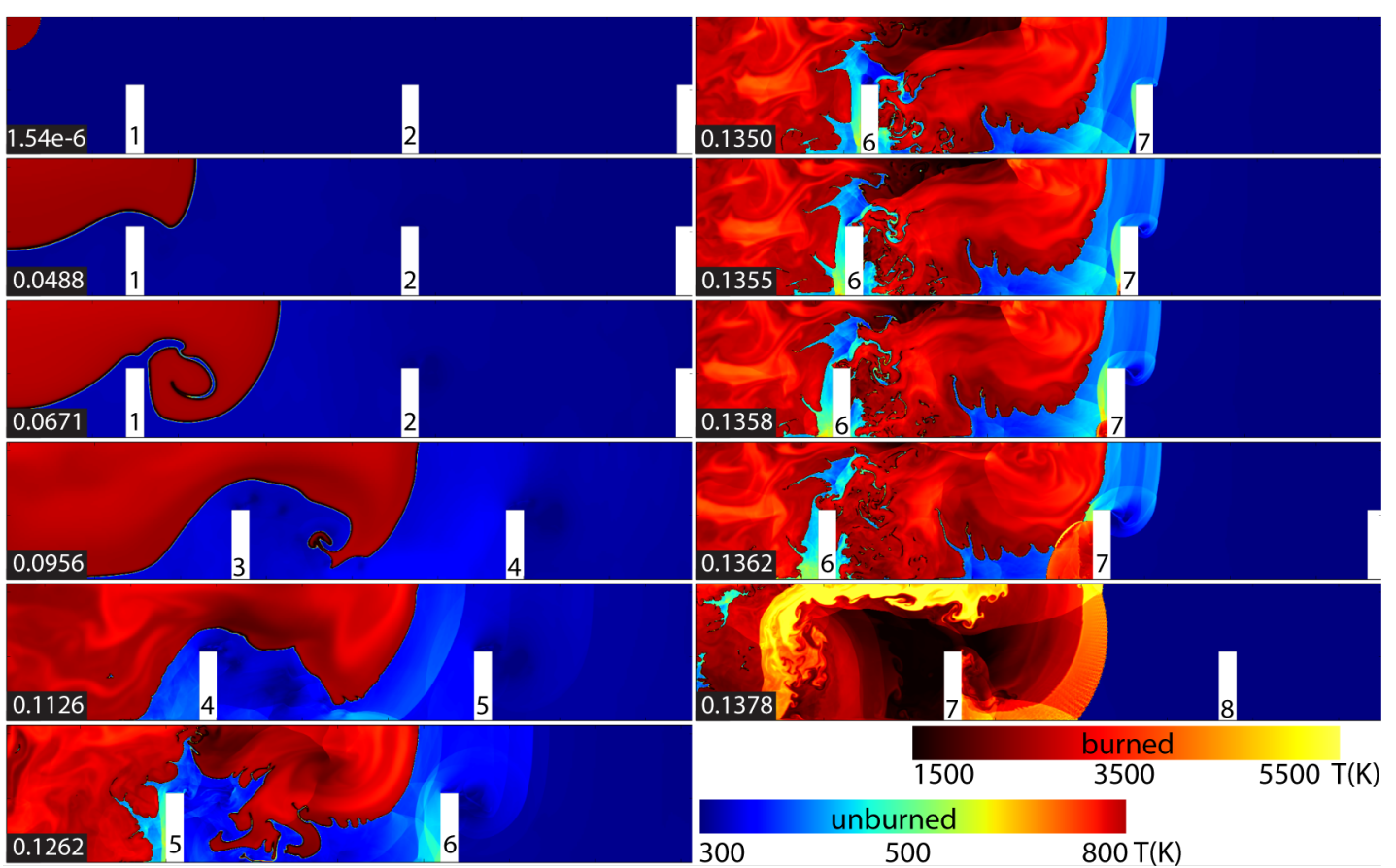

Fig. 2. Accelerating flame and detonation in $2 \mathrm{D}$ quarter-channel with $b r=0.5$. Time in milliseconds is shown in the frame corners. Obstacles are numbered.

Previous work $[11,12]$ has shown that flame acceleration is rapid in channels with $b r=0.5$ for stoichiometric mixtures of hydrogen and air. Figure 2 shows flame acceleration and transition to detonation for the stoichiometric ethylene and oxygen mixture for $b r=0.5$. As the flame passes over obstacles, its surface area increases due to expansion and fluid instabilities (Rayleigh-Taylor, RichtmyerMeshkov, and Kelvin-Helmholtz), shocks and all of their interactions. Strong leading shocks propagate into the unburned gas. The leading shock diffracts over obstacles and reflects from the channel floor, eventually forming a Mach stem $(0.1126 \mathrm{~ms})$. At $0.1355 \mathrm{~ms}$, detonation occurs in hot, unburned gas as a Mach stem reflects from an obstacle and leaves a hot spot in a gradient of reactivity. In Fig. 2, the unburned material detonates behind a Mach stem reflection from obstacle 7 at $0.1355 \mathrm{~ms}$.

When $b r$ is much smaller, 0.1 shown in Fig. 3, the flame accelerates more slowly and stays laminar 


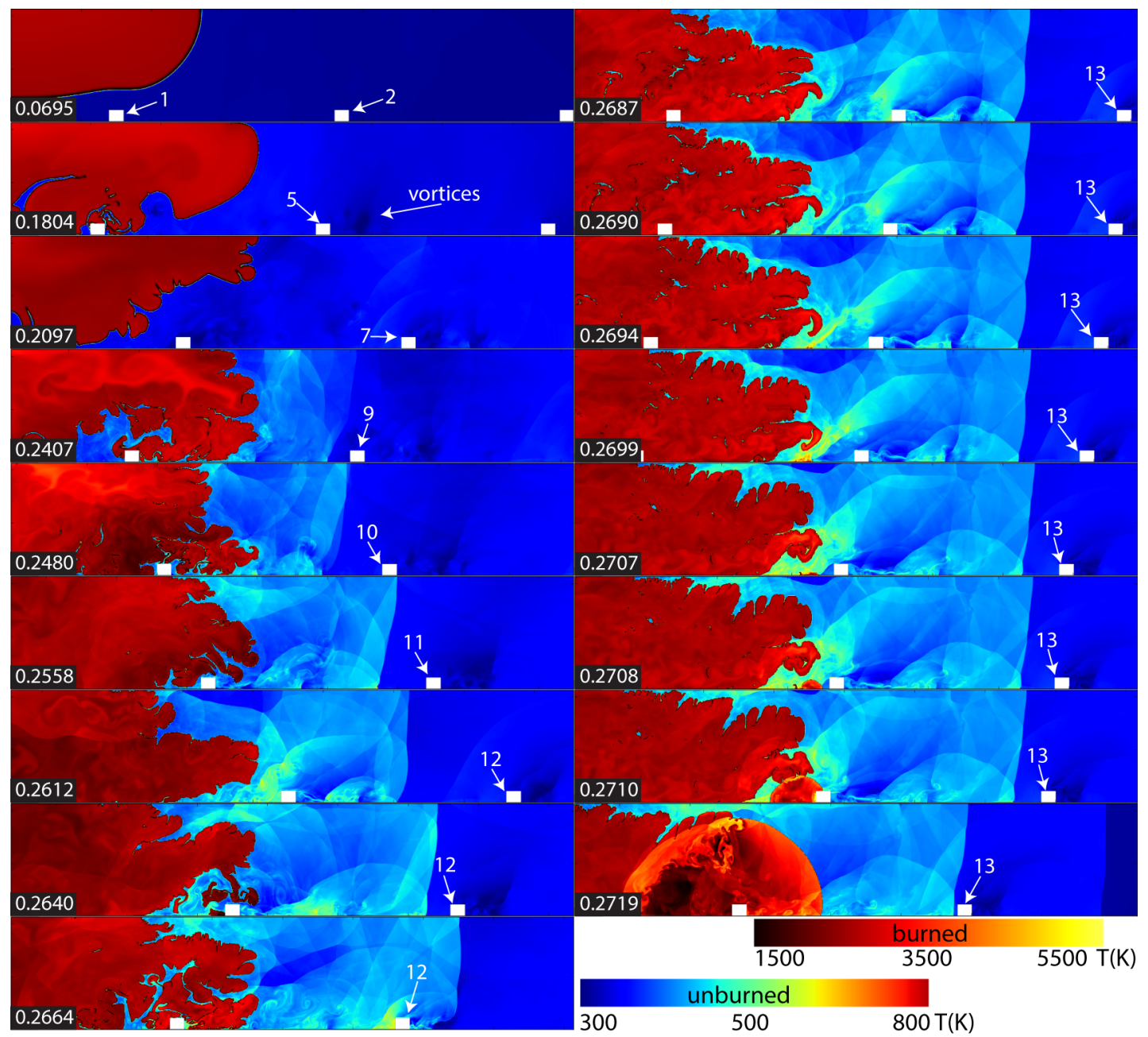

Fig. 3. Full simulation for blockage ratio of 0.10. Time in milliseconds is shown in the frame corners. Obstacles are numbered.

longer as it propagates into the channel. For $b r=0.5$, detonation occurs at $0.136 \mathrm{~ms}$ at $3.5 \mathrm{~cm}$ from the left edge, having passed six obstacles. When the $b r=0.1$, detonation occurs against the lower wall at $0.271 \mathrm{~ms}$ at $7.25 \mathrm{~cm}$, having passed 11 obstacles. Despite the major differences in time and distance to DDT, the flame development is similar in both cases. Unburned gas is driven downstream over obstacles causing vortex shedding from the obstacle's leading edges (seen starting at $0.1804 \mathrm{~ms}$ in Fig. 3). Vortices coalesce behind obstacles to form regions of recirculation. The flame surface area increases as it is pulled into the recirculation region and becomes turbulent as the acoustic waves shed by the vortices propagate through the flame. Shocks form as the flame accelerates, increasing in strength as they reflect off the obstacles, and these shocks collide with one another. Rather than seeing the rapid development of a strong leading shock that is closely coupled with the flame, as seen in Fig. 2, a relatively large unburned, turbulent region filled with shocks and strong vortices now forms between the flame and the leading shock. Figure 4 shows the complexity and dynamic structure in this shock-flame complex.

Figure 5 is an enlargement of the detonation that occurs in Fig. 3 for $b r=0.1$. Two shocks, labeled 


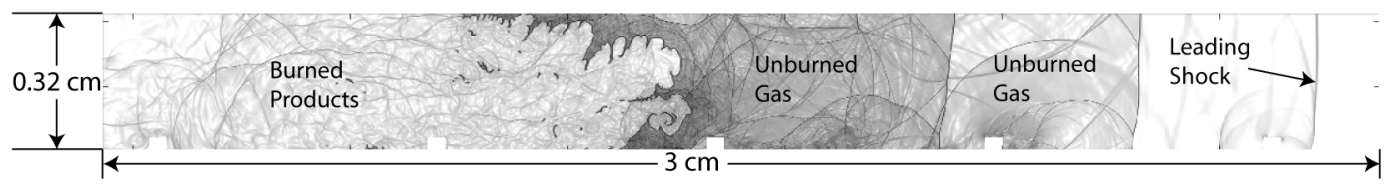

Fig. 4. Numerical schlieren showing the turbulent region.

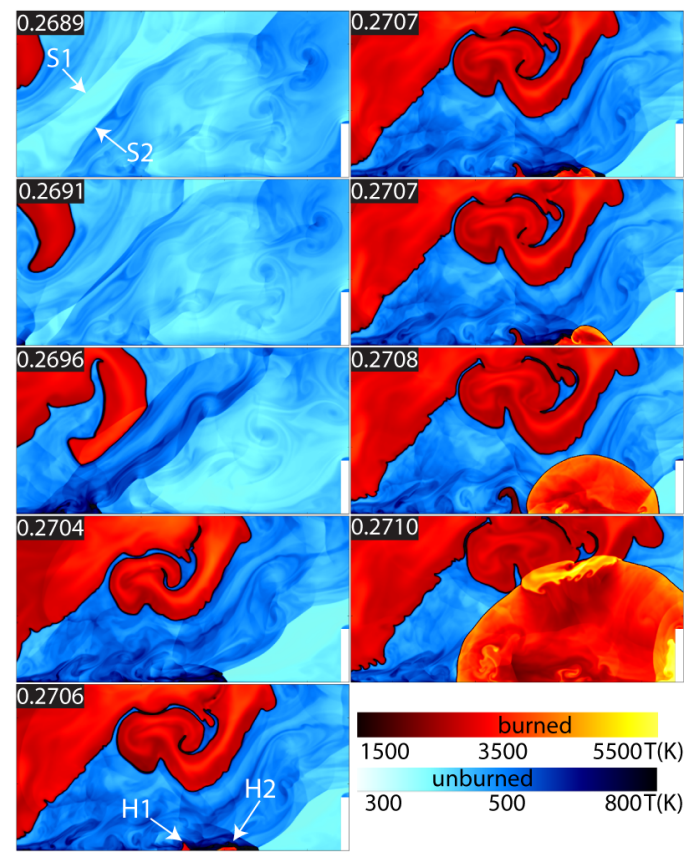

Fig. 5. Multiple shock collision and detonation. Time is shown in ms.

$\mathrm{S} 1$ and S2 at $0.2689 \mathrm{~ms}$, collide at $0.2696 \mathrm{~ms}$. Shock S1 is immediately ahead of the flame, traveling to the right, and shock $\mathrm{S} 2$ is reflected from the downstream obstacle (shown in white in the lower right corner of Fig. 5) and is traveling to the left. The shock collision ignites two hot spots in the boundary layer, labeled H1 and H2. By 0.2707 ms, H1 evolves into a flame and shock, and H2 evolves into a detonation. The analysis below shows that the confluence of shocks causes transition to detonation in hot unburned gas. This detonation mechanism has elements of both gradient ignition and direct initiation of detonation.

To quantify the change in state of unburned material compressed during the multiple-shock collision, a control volume analysis was performed on a small $33.3 \mu \mathrm{m} \times 33.3 \mu \mathrm{m}$ region at the location where $\mathrm{H} 2$ forms and becomes a detonation. The analysis tracked the change in pressure, temperature, density, and energy release rate over a $0.2 \mu$ s period from $270.5 \mu$ s to $270.7 \mu$ s. This corresponds to a time before the region is compressed by the shock collision to a time after the detonation formed and propagated through the region. Figure 6 shows the trends of the state variables. Shock compression begins at $270.55 \mu \mathrm{s}$, and the material begins to react chemically at $270.65 \mu \mathrm{s}$. The pressure and density increase dramatically, first from shock compression and then after the detonation. As the detonation propagates out of the region, the pressure and density decrease. The average temperature in the control volume 
prior to combustion is $\sim 850 \mathrm{~K}$.

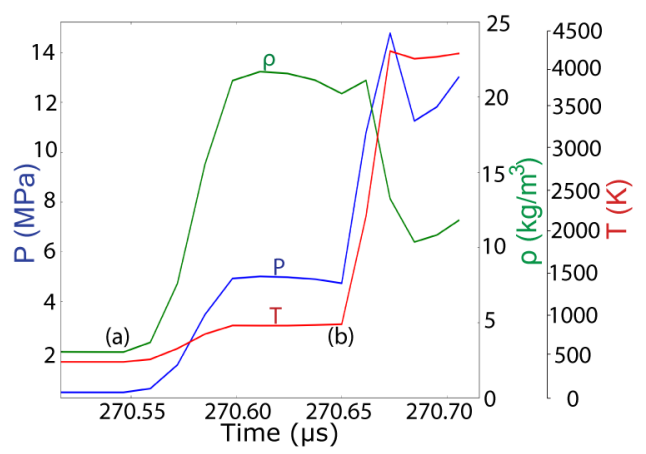

Fig. 6. State variables in the control volume from $270.5 \mu$ s to $270.7 \mu$ s (a) Shock compression (b) Detonation. The gas begins to react chemically at $270.65 \mu \mathrm{s}$.

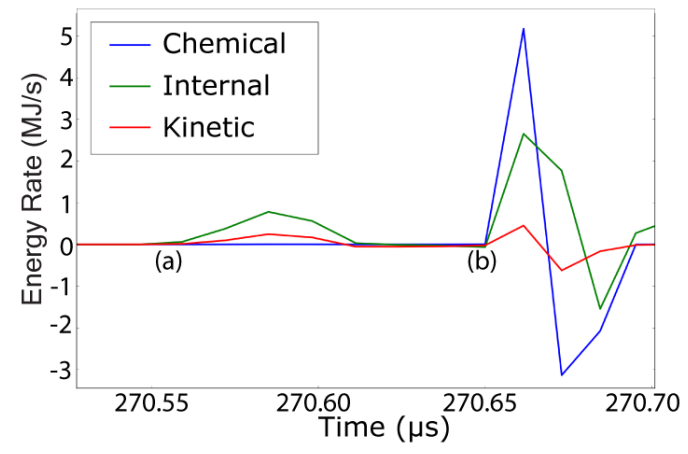

Fig. 7. Rate of energy deposition in control volume during (a) Shock compression (b) Detonation.

Figure 7 shows the rate of energy deposition into the control volume. Chemical energy is defined as heat release per $\mathrm{kg}$ of reactant, $q$, internal energy is $P /(\gamma-1)$, and kinetic energy is $0.5 \rho u^{2}$, where $u$ is the magnitude of velocity. As the material in the region is compressed, rates of internal and kinetic energy increase, followed by a second peak as the material detonates. The detonation carries energy out of the region as it propagates into the surrounding gas, resulting in the negative energy rates seen after $270.66 \mu$ s. The total energy content in the region, defined as the sum of chemical, internal, and kinetic energies, increases by a factor of 15 during shock compression.

Prior work [11] has shown that for hydrogen and air mixtures in channels of different sizes, there were different regimes of DDT. The authors found that in some of these regimes, the detonation mechanism was more sensitive to the turbulence in the flow. The variations in $b r$ described in this paper reflect the same phenomenon, that there are different regimes of DDT with varying sensitivity of the detonation mechanism to turbulence. In the $b r=0.1$ regime, the sensitivity of the solution to the turbulence causes the detonation to occur at slightly different times and locations for cases with modified initial conditions.

Figure 8 shows the detonation in another simulation with $b r=0.1$ with slightly different initial conditions. A smaller coarse mesh size of $26.7 \mu \mathrm{m}$ was used, as compared to $53.3 \mu \mathrm{m}$ in the previously 


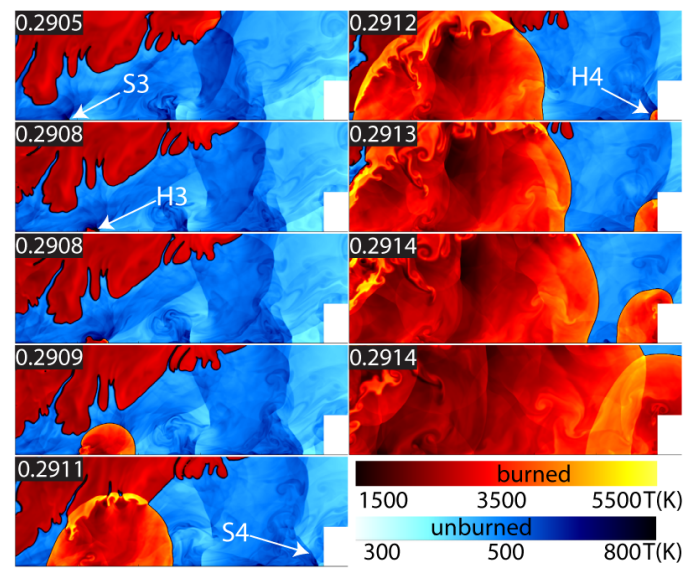

Fig. 8. Shock reflections and collisions result in initiation of two detonations. Time is shown in ms.

described $b r=0.1$ case shown in Fig. 3 . The location of the detonation is $\sim 1.2 \mathrm{~cm}$ further along the channel boundary than the detonation that occurred in Fig. 5, however, it again appears where shock collisions and reflections are focused. The shock immediately ahead of the flame, labeled S3, reflects off the channel boundary and ignites a hot spot $\mathrm{H} 3$ in the boundary layer. The hot spot becomes a detonation while another shock ahead of the flame, labeled S4, reflects off the obstacle shown in the lower right corner of the figure. The reflection of S4 ignites another hot spot, labeled H4, that also becomes a detonation. The two detonations combine and propagate down the channel. Although the time and location of detonation is stochastic due to turbulence in the flow, which is heavily dependent on initial conditions such as grid size, the detonation mechanism in the low br regime is robust. This is consistent with previous work investigating the stochasticity of DDT in flow regimes that are sensitive to turbulence in the flow [11].

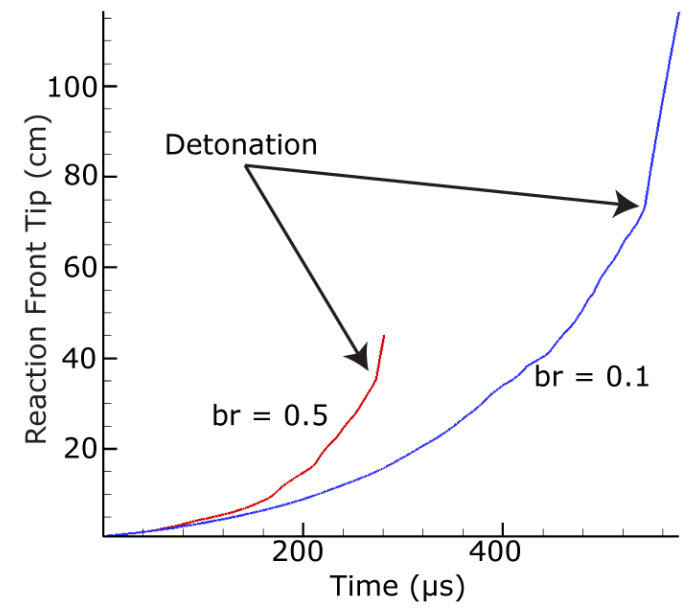

Fig. 9. Forward position of the reaction front within the channel as a function of time for 0.1 and 0.5 blockage ratio cases. Detonations occur at inflection points on the curves.

A comparison of the simulations with $b r=0.5$ and 0.1 shows that obstacle size plays a major role in the flame acceleration, turbulence in the flame and unburned gas, shock formation, time or distance 
to detonation, and detonation mechanism. Figure 9 shows the position of the tip of the reaction front as a function of time for both blockage ratios. Higher values of $b r$ result in a more predictable flow as strong leading shocks form very close to the flame. These obstacles consistently produce Mach-stems and detonations that form behind Mach-stem reflections. For this stoichiometric ethylene and oxygen mixture, the flame never falls more than $\sim 0.2 \mathrm{~cm}$ behind the leading shock. When $b r$ decreases, the leading shocks form as far as $1.5 \mathrm{~cm}$ ahead of the flame tip, creating a large, unburned turbulent region ahead of the flame. Rather than the detonation always occurring in a Mach reflection against an obstacle, they may occur anywhere that energy is focused sufficiently to initiate the detonation. The actual detonation location is stochastic because the turbulent region is filled with shock collisions and reflections that may focus sufficient energy to detonate the unburned gas anywhere within the turbulent region ahead of the flame.

Turbulence, an essentially stochastic process, dominates this region of shock collisions, shockboundary layer interactions, and fluid instabilities. This is complicated even further by the nonequilibrium nature of the turbulence itself in these scenarios.

\section{Conclusions}

Multidimensional, unsteady numerical simulations of an initially homogeneous mixture of ethylene and oxygen in partially blocked channels were performed to determine how the mechanism of DDT changes as the blockage ratio $(b r)$ is decreased. This paper compared two cases, $b r=0.5$ and 0.1 .

For $b r=0.5$, detonation occurs due to the creation of a hot spot in a gradient of reactivity that forms behind a Mach reflection [11-13]. This was shown for a different fuel-oxidizer mixture than used in previous work. In the case with $b r=0.1$, shock collisions increase the temperature, pressure, and density of the unburned material. These effects and interactions occur frequently due to the complexity of the shock structures and interactions in the unburned turbulent region between the flame and the leading shock. As the flame propagates down the channel, the strength of the shock collisions and reflections increases in this region. Multiple shocks collide and reflect against the channel boundary, focusing energy in a localized region of unburned material and detonating the mixture. These results are consistent with direct initiation theory [16] and with detonations seen in experiments using similar blockage ratios $(b r<0.3)[22]$. Sufficient energy is deposited into a localized region on a timescale that is small relative to the acoustic timescale of the gas, initiating a detonation.

An additional simulation, with $b r=0.1$, investigated the sensitivity of the solution to initial conditions. Modifying the coarse mesh size resulted in a difference in detonation time and location of 0.02 $\mathrm{ms}$ and $1.2 \mathrm{~cm}$, respectively, but the detonation mechanism was the same. This phenomenon was also 
seen in previous work with a different fuel-oxidizer mixture [11], where it was determined that some regimes of DDT are especially sensitive to turbulence in the flow, which is heavily dependent on initial conditions.

It is important to understand the similarities and differences in flame acceleration and DDT with different fuel-oxidizer mixtures. The small detonation cell size, fast reactions, and rapid energy release in the combustion of ethylene-oxygen result in a smaller length scale than in previous work with other mixtures [11-13], however, the detonation mechanism for the $b r=0.5$ case in previous work with other mixtures is the same as what is observed for the $b r=0.5$ case for ethylene-oxygen. Future work to directly compare results from different mixtures, such as where and when DDT occur, the flow structures that form ahead of the turbulent flame, and whether the flame is galloping or fast, will be a useful contribution to the study of DDT in channels.

\section{Acknowledgments}

This work was supported by the Office of Naval Research, the University of Maryland through Minta Martin Endowment Funds in the Department of Aerospace Engineering, and the Glenn L. Martin Institute Chaired Professorship at the A. James Clark School of Engineering. The computations were carried out on University of Maryland supercomputing resources (http://www.it.umd.edu/hpcc). The authors thank David Kessler and Fokion Egolfopoulos for their assistance in developing the chemical model used in these simulations, as well as Vadim Gamezo for sharing his insights on DDT in channels with obstacles. The authors particularly acknowledge the help of Alp Ozgen in determining the optimal chemical-diffusive model.

\section{References}

[1] P. Middha, O. Hansen, Process Safety Progress 27 (2008) 192-204.

[2] P. Thibault, L. Britton, F. Zhang, Process Safety Progress 19 (2000) 125-139.

[3] R. Sorin, R. Zitoun, D. Desbordes, Shock Waves 15 (2006) 137-145.

[4] R. Smirnov, R. Zitoun, D. Desbordes, Journal of Engineering Physics and Thermophysics 83 (2010) $1287-1316$.

[5] Y. Huang, H. Tang, J. Li, J. Wang, Journal of Aerospace Engineering 225 (2011) 441-448.

[6] R. Zipf, V. Gamezo, M. Sapko, W. Marchewka, K. Mohamed, E. Oran, D. Kessler, E. Weiss, J. Addis, F. Karnack, D. Sellers, Journal of Loss Prevention in the Process Industries 26 (2013) 295-301.

[7] J. Lee, A. Higgins, Phil. Trans. R. Soc. Lond. 357 (1999) 3503-3521.

[8] B. Zhang, H. Ng, J. Lee, Journal of Loss Prevention in the Process Industries 26 (2013) 1293-1299. 
[9] J. Lee, The Detonation Phenomenon, Cambridge University Press, New York, NY, 1 edition, 2008.

[10] E. S. Oran, V. N. Gamezo, Combust. Flame 148 (2007) 4-47.

[11] V. N. Gamezo, T. Ogawa, E. S. Oran, Combust. Flame 155 (2008) 302-315.

[12] V. N. Gamezo, T. Ogawa, E. S. Oran, Proc. Combust. Inst. 31 (2007) 2463-2471.

[13] D. A. Kessler, V. N. Gamezo, E. S. Oran, Combust. Flame 157 (2010) 2063-2077.

[14] G. Ciccarelli, C. Fowler, M. Bardon, Shock Waves 14 (2005) 161-166.

[15] A. Na'inna, H. Phylaktou, G. Andrews, International Symposium on Safety Science and Technology 84 (2014) 306-319.

[16] A. D. Kiverin, D. R. Kassoy, M. F. Ivanov, M. A. Liberman, Phys. Rev. E 87 (2013) 033015.

[17] D. Kassoy, Journal of Engineering Mathematics 68 (2010) 249-262.

[18] Y. Zeldovich, V. Librovich, G. Makhviladze, G. Sivashinsky, Astronautica Acta 15 (1970) 313-321.

[19] J. Lee, R. Knystautas, N. Yoshikawa, Acta Astronautica 5 (1978) 971-982.

[20] G. Ciccarelli, S. Dorofeev, Progress in Energy and Combustion Science 34 (2008) 499 - 550.

[21] V. N. Gamezo, T. Ogawa, E. S. Oran, AIAA Paper 2009-440 (2009).

[22] S. Maeda, S. Minami, D. Okamoto, T. Obara, Visualization of Deflagration-to-detonation Transitions in a Channel with Repeated Obstacles, Proc. of the 25th Int. Colloquium on the Dynamics of Explosions and Reactive Systems, 2015.

[23] R. W. Houim, K. K. Kuo, J. Comput. Phys. 230 (2011) 8527-8553.

[24] Center for Computational Sciences and Engineering, University of California, Berkeley, Boxlib, Retrieved 03-2015.

[25] M. Wu, W. Kuo, Proc. of the Combust. Inst. 34 (2013) 2017-2024. 


\section{List of Figures and Captions}

Figure 1: Computational setup in units of $\mathrm{cm}$. Obstacles evenly spaced along channel length $L$. Radius of the initial burned region is $0.08 \mathrm{~cm}$. Blockage ratio is 0.1 in this case.

Figure 2: Accelerating flame and detonation in 2D quarter-channel with $b r=0.5$. Time in milliseconds is shown in the frame corners. Obstacles are numbered.

Figure 3: Full simulation for blockage ratio of 0.10. Time in milliseconds is shown in the frame corners. Obstacles are numbered.

Figure 4: Numerical schlieren showing the turbulent region.

Figure 5: Multiple shock collision and detonation. Time is shown in ms.

Figure 6: State variables in the control volume from $270.5 \mu \mathrm{s}$ to $270.7 \mu \mathrm{s}$ (a) Shock compression (b) Detonation. The gas begins to react chemically at $270.65 \mu \mathrm{s}$.

Figure 7: Rate of energy deposition in control volume during (a) Shock compression (b) Detonation.

Figure 8: Shock reflections and collisions result in initiation of two detonations. Time is shown in ms.

Figure 9: Forward position of the reaction front within the channel as a function of time for 0.1 and 0.5 blockage ratio cases. Detonations occur at inflection points on the curves.

Table 1: Input model parameters and output combustion wave properties for stoichiometric ethylene and oxygen initially at $1 \mathrm{~atm}$ and $298 \mathrm{~K}$. 